\title{
Feuilleton.
}

\section{Die pathologische Physiologie an den Universitäten Oesterreich-Ungarns.}

Von Privatdozent Dr. Adolf Bickel, Assistenten am Königl. pathologischen Institut in Berlin.

Die von Rudolf Virchow geplante und von J. Orth weiter ausgestaltete "Experimentell-biologische Abteilung“ in dem Neubau des Königl. Pathologischen Instituts zu Berlin bedeutet eine Einrichtung, die wir bis jetzt in Deutschland nicht besitzen. Diese Abteilung entspricht nämlich den Instituten für "allgemeine und experimentelle Pathologie“ des Auslandes, den Pflegestätten der pathologischen Physiologie. Mit der Einrichtung dieser Abteilung am Berliner Pathologischen Institut wird auch bei uns der Anfang gemacht werden, der pathologischen Physiologie in ihrem ganzen Umfange die ihr gebührende Stellung im Rahmen der medizinischen Fakultät $\mathrm{zu}$ erringen, indem das Berliner Institut nun fortan neben seiner schon lange bestehenden Abteilung für pathologische Chemie auch über eine solche verfügt, die dem anderen Teil, wenn man will, dem "speziell pathologisch-physiologischen" Abschnitte der pathologischen Physiologie geweiht ist.

Das Land, in dem sich die pathologische Physiologie zuerst zu einem selbständigen Lehr- und Forschungsgebiete entwickelt hat, ist Oesterreich-Ungarn. Auf den Vorschlag Rokitanskys wurde in Wien von der österreichischen Regierung im Jahr 1868 das erste derartige Laboratorium der Welt errichtet und Stricker zum Professor der Experimentalpathologie ernannt. Stricker war Sekundärarzt am k. k. allgemeinen Krankenhause, dann Privatdozent für Entwicklungsgeschichte, Assistent an der physiologischen Lehrkanzel unter Brücke und schlieflich klinischer Adjunkt 0 ppolzers gewesen und vereinigte so bei diesem für die damalige Zeit nicht gewöhnlichen Gange der Ausbildung die physiologische mit der klinischen Schulung, weshalb Rokitansky ihn für besonders geeignet hielt, die von ihm inaugurierte Disziplin auszubauen. Nach dem Wiener Muster wurden dann in den folgenden. Jahren entsprechende Institute an den übrigen Universitäten der Monarchie eingerichtet, und das Ausland beeilte sich, dem von Oesterreich gegebenen Beispiele nachzueifern.

Es ist um so mehr zu verwundern, daf Deutschland in der Ausgestaltung der pathologischen Physiologie die Führung der Wiener Schule überlassen hat, als bereits im Jahre 1846 Vir chow mit seiner ganzen Autorität für die Bedeutung der pathologischen Physiologie eingetreten ist. Er schrieb damals: "- - Es scheint mir daher ebenso richtig als sachgemäß zu sein, daß man daran denke, eine Wissenschaft zu begründen, die gleich der univer- 
sellen Anatomie und Physiologie als pathologische Anatomie und Physiologie an die Stelle der allgemeinen Pathologie trete. - Die pathologische Physiologie kann niemals aus der pathologischen Anatomie konstruiert werden. Die pathologische Physiologie hat nur zwei Wege, einen unvollkommenen, den der klinischen Beobachtung, und einen möglichst vollkommenen, den des Experiments. Sie ist daher kein Machwerk der Spekulation, der Hypothese, der Willkür oder der Ueberzeugung, keine aus der pathologischen Anatomie abgeleitete Disziplin, sondern eine große, selbständige und souveräne Wissenschaft der Tatsachen und Experimente." Indem man sich in Oesterreich gewissermaßen diese Auffassung der Dinge zu eigen machte, hat man es dort gleichzeitig verstanden, die praktischen Konsequenzen aus ihr zu ziehen und ihnen im vollsten Umfange gerecht $\mathrm{zu}$ werden.

Eine Studienreise, welche mich während des verflossenen Wintersemesters nach Oesterreich-Ungarn führte, gab mir Gelegenheit, die dortigen Institute für diese Disziplin zu besuchen und einen Einblick zu tun in den Lehrbetrieb, wie er an ihnen gehandhabt wird. Ich lernte so die Institute in Wien, Budapest, Graz, Innsbruck und Prag. kennen und halte es um so mehr für angezeigt, über meine Reise-Eindrücke an dieser Stelle zu berichten, als bei uns in Deutschland und speziell auch in Preußen die pathologische Physiologie als eigener Lehrgegenstand an der Universität noch eine Fremde ist, die sich ihr Bürgerrecht erst erwerben soll.

Die selbständige Stellung, welche die pathologische Physiologie in Oesterreich-Ungarn einnimmt, dokumentiert sich nicht nur darin, daß ihr eigene Institute und ordentliche Professuren an allen Universitäten zu Gebote stehen, sondern ihre Gleichordnung mit den anderen Disziplinen der Medizin spricht sich auch darin aus, daß sie einen besonderen Prüfungsgegenstand in dem ärztlichen Staatsexamen darstellt.

Die Institute, in denen die pathologische Physiologie getrieben wird, bezeichnete man in Oesterreich mit dem etwas unglücklich gewählten Namen, über den auch heute noch Unzufriedenheit herrscht: „Institute für allgemeine und experimentelle Pathologie“. Denn was in ihnen bearbeitet und was in ihnen gelehrt werden soll, ist die pathologische Physiologie, die Lehre von den Vorgängen im lebenden Körper während des kranken Zustandes und den Erscheinungen, die diese zeitigen, die pathologische Physiologie, deren Aufgabe es ist, die Variationen darzustellen, die das Geschehen im Körper unter dem Eindruck bestimmter abnormer Bedingungen erfährt.

Die österreichisch-ungarische Regierung hat es sich angelegen sein lassen, alles das zu realisieren, was an Instituten und sonstigen Einrichtungen sich als notwendig erwies, um einen geordneten Lehr- und Forschungsbetrieb in dieser Disziplin an den Universitäten zu ermöglichen.

In Wien feierte die pathologische Physiologie, beziehungsweise experimentelle Pathologie unter Stricker ihre größten Triumphe. Das Laboratorium, welches Stricker im Jahre 1868 erhielt, erwies sich bald als zu klein und den Ansprüchen, die der Unterricht und die Forschung an es stellten, nicht mehr gewachsen. Als im Jahre 1883 dann das Pathologische Institut, das gleichzeitig das Laboratorium für medizinische Chemie, für gerichtliche Medizin und für allgemeine und experimentelle Pathologie neben der pathologisch-anatomischen Abteilung beherbergte, durch einen Umbau eine bedeutende Erweiterung erfahren hatte, erhielt das Institut für experimentelle Pathologie neben einer größeren Zahl von Arbeitsräumen einen eigenen Hörsaal zugewiesen, der zirka 400 Personen aufnehmen konnte. Die Bibliothek wurde mit der der anderen Institute des Gebäudes vereinigt. Weiterhin baute man Tierställe, und in neuester Zeit ist dem Institut dann noch ein aseptisches Operationszimmer zugeteilt worden. An dem Wiener Institut für experimentelle $\mathrm{Pa}$ thologie werden heute zwei Assistenten und zwei Diener beschäftigt; die Jahresdotation beträgt zirka 1800 Gulden. Indessen hat sich das Institut auch in dieser Ausdehnung als unzulänglich erwiesen und es ist ein Neubau vorgesehen.

Die Einrichtungen, die das Wiener Institut für den Unterricht besitzt und die es vor allem der Fürsorge Strickers verdankt, sind weitberühmt und verdienen bei jeder Neueinrichtung eines derartigen Instituts die sorgfältigste Berücksichtigung. Vor allem erwähne ich hier den Apparat für epidiaskopische Projektionen, der einen so weiten Raum für die Aufnahme der Objekte hat, daß in ihm nicht nur größere Tiere, wie Kaninchen und Hunde, untergebracht werden können, sondern daß es sogar möglich ist, in ihm an derartigen Tieren beliebige Eingriffe zu experimentellen Zwecken zu vollziehen. So können alle Manipulationen, selbst größere Operationen unter aseptischen Kautelen in ihm vorgenommen und in ihrem ganzen Verlauf auf den Schirm projiziert und für das Auditorium sichtbar gemacht werden. Bemerkenswert ist ferner das sogenannte durchsichtige Kymographion. Kurven aller Art von Menschen und Tieren werden hierbei anstatt auf eine berufte Trommel auf eine Glasscheibe aufgezeichnet, die ein Uhrwerk an dem Projektionsapparat vorüberführt. Auf dem Projektionsschirm sieht der Student, wie sich die Kurve aufzeichnet, und er kann bei der enormen Bildgröße all ihre Feinheiten aufs genaueste verfolgen. Ferner ist in den Hörsaal von dem maschinell getriebenen Apparat für künstliche Atmung, der sich in einem anstoßenden Arbeitsraum befindet, eine Leitung gelegt, so daß alle Versuche, bei denen eine künstliche Ventilation der Tiere erforderlich ist, leicht während der Vorlesung sich ausführen lassen.

Dieser sorgfältigen Ausgestaltung des Unterrichts, von der ich nur einige wenige Punkte hier hervorheben konnte, ist es nicht zum letzten zuzuschreiben, daß Stricker in den 90 er Jahren öfters eine Zuhörerschaft von 600-800 Köpfen hatte. Der große Hörsaal des Instituts erwies sich als viel zu klein, und Stricker mußte seine Vorlesung doppelt halten, um einem so enormen Andrang von Hörern gerecht werden zu können.

Was nun die eigentlichen Laboratoriumsräume des Instituts angeht, so habe ich schon hervorgehoben, daß ihnen in neuester Zeit ein aseptisches Operationszimmer zugefügt worden ist, das erst die Ausführung einer großen Reihe von Untersuchungen ermöglicht hat, die vorher ohne diesen Raum undurchführbar waren. Die Einrichtung dieses Zimmers ist genau nach dem Muster der Operationssäle in chirurgischen Kliniken getroffen. Im übrigen sehen wir das Wiener Laboratorium reich ausgestattet mit allen den mannigfaltigen Apparaten und Vorrichtungen, deren ein Experimentalinstitut bedarf, wofern in ihm sich eine fruchtbare Tätigkeit in der Forschung entfalten soll. Das Wiener Institut ist eine Arbeitsstätte für Aerzte und Studenten, und es darf heute, wie zu Strickers Zeiten, als eine Zentrale für die wissenschaftliche Arbeit in der Wiener medizinischen Fakultät angesprochen werden.

In Budapest hat man ein großes, mehrstöckiges Institut für experimentelle Pathologie neuerdings errichtet. Es soll während dieses Sommers bezogen werden. Ihm ist angegliedert das Laboratorium für die Herstellung des Schutzstoffes zur Imıfung tollwutverdächtiger Menschen. Ein neues Krankenhaus mit zirka 100 Betten ist dem Institut gleichfalls zugeteilt; allerdings finden dort lediglich Personen Aufnahme, die sich der Schutzimpfung unterzogen haben.

Von dem neuen Budapester Institut für experimentelle Pathologie, das ich leider im Betrieb noch nicht sehen konnte, vermag ich nur so viel zu sagen, daß der Bau mit allem erdenklichen Luxus und Raffinement ausgestattet ist und daß es nach dem geradezu pompösen neuen Physiologischen Institut in Wien wohl das schönste ist, das Oesterreich-Ungarn zurzeit besitzt. Ich will noch hervorheben, daß sich in ihm eine besondere Abteilung für Bakteriologie mit einem eigenen Abteilungsvorsteher befinden wird. Das alte Budapester lnstitut ist klein und mit anderen Laboratorien zusammen in einem Stockwerk eines größeren Gebäudes der Universität untergebracht.

In dem großen und modernen Institut, das für allgemeine und experimentelle Pathologie in Graz vor wenigen Jahren neu gebaut wurde, begegnen wir beim Unterricht, der in Graz in mustergiltiger Weise ausgestaltet ist, genau wie in Wien einer ausgiebigen Anwendung. der Projektionsmethoden. Ich habe dort unter anderem die Vorgänge bei der Blutstauung an der Schwimmhaut des lebenden Frosches in der Projektion gesehen und muß sagen: das war alles so plastisch, klar und eindrucksvoll in der vieltausendfältigen Vergrößerung, bei der man die Blutkörperchen wie große Scheiben sieht, die sich da bewegen, aneinanderpressen und durch das Gefäßrohr drängen, daß man dies Bild und die ganzen Vorgänge nimmermehr vergessen kann, wenn man es einmal gesehen hat.

Das Grazer Institut zeichnet sich aus durch die großen, hellen und luftigen Laboratoriumsräume, die sich wie eine Flucht von Sälen aneinander reihen. Das Institut ist mit einem reichen Instrumentarium versehen, und darunter verdienen besonders die 
sinnreichen Apparate von Prof. Klemensiewicz, die zur Fixation und Kühlung der lebenden Objekte bei der Projektion dienen, Beachtung. Eine grobe Sorgfalt ist ferner in Graz auf den Bau der Tierställe des Instituts verwandt worden. In dem Institut befindet sich weiterhin ein Saal eigens für bakteriologische Zwecke eingerichtet. Zurzeit sind zwei Assistenten und zwei Diener an dem Grazer Institut angestellt.

Das Laboratorium für allgemeine und experimentelle Pathologie in Innsbruck ist mit dem Prager das kleinste in Oesterreich. Die Arbeitsräume in beiden Instituten sind wenig zahlreich und meist nicht grob. Sie genügen, wenigstens in Innsbruck, kaum den Ansprüchen, die Unterricht und Forschung an sie stellen. Es ist indessen nur eine Frage der Zeit, daß auch hier durch Neubauten Abhilfe geschaffen wird, zumal besonders auch die Hörsäle in Innsbruck und Prag mangelhaft sind, da ihnen eine dem speziellen Zwecke angepaßte Ausstattung gänzlich fehlt. Man hat das alte Innsbrucker Institut jüngst insofern etwas verbessert, als man in ihm einen zweifenstrigen Arbeitsraum als bakteriologisches Laboratorium nach modernem Muster einrichtete. Dieser Raum muß jedoch gleichzeitig als Operationszimmer benutzt werden und ferner dient er zur Aufnahme des Projektionsapparates. Das erhellt genügend den Platzmangel, der im Innsbrucker Institut zur Zeit herrscht. Daf sich dort der Institutsdiener mit seiner Werkstätte im Korridor einrichten mußte, kann den Besucher des Instituts nach alledem nicht Wunder nehmen. Auf der anderen Seite hat die österreichische Regierung dem Institute auf seine berechtigte Forderung außer der Einrichtung des bakteriologischen Laboratoriums insofern noch eine weitere Abschlagszahlung bewilligt, als sie ihm Tierställe baute, die allen Anforderungen der heutigen Zeit entsprechen.

In Prag liegen die Verhältnisse immerhin noch günstiger als in Innsbruck. Im Prager Institut fällt vor allem eine reiche instrumentelle Ausstattung auf; insonderheit sind alle die Apparate und Einrichtungen, deren man zur Demonstration und zu Untersuchungen über die pathologische Physiologie des Kreislaufs und des Herzens bedarf, dort in großer Vollständigkeit vorhanden; ein Teil der Apparate ist nach den Angaben von Prof. Hering angefertigt. Was das Prager Institut für experimentelle Pathologie aber vor allen anderen in Oesterreich auszeichnet, ist der Umstand, daß ihm eine kleine Krankenabteilung beigegeben ist, die über ein nach den jeweiligen Bedürfnissen des Instituts ausgewähltes Krankenmaterial verfügt. Die Abteilung ist in einem Pavillon des benachbarten allgemeinen Krankenhauses untergebracht und besteht aus zwei Krankensälen, einem Isolierzimmer, einem Zimmer für den Chef des Instituts, einem Laboratorium und einigen kleinen Nebenräumen. Diese Krankenabteilung, die schon unter Knoll geschaffen wurde, befindet sich in einem Neubau und ist in jeder Beziehung elegant ausgestattet.

Die Angliederung einer solchen Abteilung an ein Institut für experimentelle Pathologie, wie es in Prag durchgeführt ist, hat sich sowohl für den Unterricht als auch für die Forschung als in höchstem Grade segensreich erwiesen. Es ist nämlich dadurch die Möglichkeit gegeben, einzelne Symptome, die in der theoretischen Vorlesung durchgesprochen und am Tier experimentell vor den Augen der Studenten erzeugt worden sind, nun auch am kranken Menschen zu demonstrieren, und es wird so in einer ebenso instruktiven wie vollkommenen Weise dem jungen Mediziner, ehe er in die Klinik eintritt, gezeigt, wie die Erscheinungen, die er am Krankenbette später in großer und wechselvoller Fülle sehen wird, sich ableiten lassen aus bestimmten Alterationen der Organfunktionen, deren Kenntnis er hinwieder aus der normalphysiologischen Vorlesung mitbringt; es können so, wenn dem pathologischen Physiologen Krankenmaterial zur Verfügung steht, in einer systematischen Weise vor den Studenten beim Unterricht die Brücken von der normalen Physiologie zur Klinik geschlagen werden; und damit wird eben voll und ganz das erreicht, was die Vorlesung über pathologische Physiologie bezwecken soll.

Ich darf hier anfügen, daß ich selbst in Göttingen, wo mir das Material der medizinischen Klinik von Herrn Geh. Med.-Rat Prof. Dr. Ebstein in liberalster Weise für meine Vorlesungen über pathologische Physiologie zur Verfügung gestellt worden war, immer wieder habe erfahren können, wie gerade die Demonstration der Erscheinungen am kranken Menschen, die in der Vorlesung erst theoretisch besprochen, dann den Hörern im Experiment vorgeführt worden waren, nicht nur die Aufmerksam- keit der Studenten in besonderem Maße fesselte, sondern wie ihnen eben diese Behandlung des Stoffes oft erst das rechte Verständnis für die klinische Symptomatologie erschloß und sie bei der Beurteilung ganzer Krankheitsbilder in den rein klinischen Kursen zu physiologischem Denken anhielt.

Für die Forschung in einem Institut für experimentelle Pathologie hat sich nach den in Prag gesammelten Erfahrungen die Beigabe einer Krankenabteilung als nicht minder wertvoll erwiesen. Einmal sichert der stete Konnex mit dem kranken Menschen, daf die Probleme, die in dem Institute zu bearbeiten sind, auch stets mit Rücksicht auf die Bedürfnisse der Klinik gestellt werden. Die pathologisch-physiologische Forschung wird so bewahrt vor einer Loslösung von der klinischen Medizin; eine solche Entfremdung könnte weder dieser noch jener zum Vorteil gereichen. Ferner aber ist es möglich, wenn dem Laboratorium für pathologische Physiologie eine kleine Krankenabteilung zur Verfügung steht, einen steten Vergleich zu ziehen zwischen den Ergebnissen des Tierversuchs und den Erscheinungen am Krankenbette. Und gerade in diesem Sinne wird auch in Prag die Krankenabteilung des Instituts in reichstem Maße ausgenutzt.

Was nun die Handhabung des Unterrichtes in der pathologischen Physiologie in Oesterreich-Ungarn anlangt, so ist es begreiflich, daß bei einem derartig ausgedehnten Gebiete, wie es das der pathologischen Physiologie ist, keine absolute Uebereinstimmung in der Behandlung des Stoffes in der Vorlesung an den verschiedenen Universitäten besteht. Insonderheit differieren die einzelnen Lehrer insofern, als sie in verschiedener Weise die Lehre von der Aetiologie-der Krankheiten mit in den Bereich ihrer Vorlesung hineinziehen, die nach meiner Meinung, streng genommen, in einem Kolleg über pathologische Physiologie höchstens in ihren allgemeinen Umrissen dargestellt werden darf.

Daß außerdem an den verschiedenen Hochschulen auch die einzelnen Kapitel aus der pathologischen Physiologie selbst nicht völlig gleichmäßig behandelt werden, ist verständlich, da hier, wie bei allen anderen theoretischen Fächern, naturgemäß die jeweiligen Interessen und Neigungen des Lehrers einen bestimmenden Einfluß auf die Gestaltung der Vorlesung ausüben.

Insofern aber besteht eine schöne Harmonie in der Handhabung des Unterrichts in der pathologischen Physiologie an sämtlichen Hochschulen Oesterreich-Ungarns, als überall dieses Kolleg als eine Experimentalvorlesung in großem Stile gehandhabt wird. Darauf ist auch die Institutseinrichtung allüberall zugeschnitten: die Hilfsmittel an Apparaten sind reiche, und den Instituten steht ein geschultes Hilfspersonal zur Verfügung, ohne das natürlich die Durchführung einer derartigen Experimentalvorlesung illusorisch sein würde.

So sehen wir in Oesterreich-Ungarn an allen Hochschulen ein frisch pulsierendes Leben in einer Disziplin der medizinischen Wissenschaft, die wir in Deutschland als ein selbständiges Lehrund Forschungsgebiet noch nicht kennen. Dort hat man ihr schon vor Jahrzehnten an jeder Universität ein eigenes Heim bereitet, in dem sie sich entwickeln sollte und über das sie hinausgewachsen ist. Den gesteigerten Ansprüchen nachkommend, hat die österreichisch-ungarische Regierung in ihrem Lande der pathologischen Physiologie neue Institute mit glänzend ausgestatteten Laboratorien errichtet oder ist dabei, wo die alten noch stehen, diese durch Neubauten zu ersetzen.

In liebenswürdigem Entgegenkommen hat man mir in Oesterreich-Ungarn die Institute fül allgemeine und experimentelle Pathologie gezeigt und mir ihre Einrichtungen vorgeführt; man hat mir aber auch so manches Mal seine Freude darüber ausgedrückt, daß man im Interesse der pathologischen Physiologie nun auch bei uns den Anfang mache, mit der Rückständigkeit aufzuräumen, in der Preußen und Deutschland sich bis heute hinsichtlich der Einrichtung dieses Faches an der Universität befunden haben.

Ich will nicht verfehlen, den Herren Professoren Biedl, Hering, Högyes, Klemensiewicz, Löwit, Paltauf und Székély an dieser Stelle nochmals für die freundschaftliche und herzliche Aufnahme zu danken, die ich in ihren Instituten erfahren habe. 